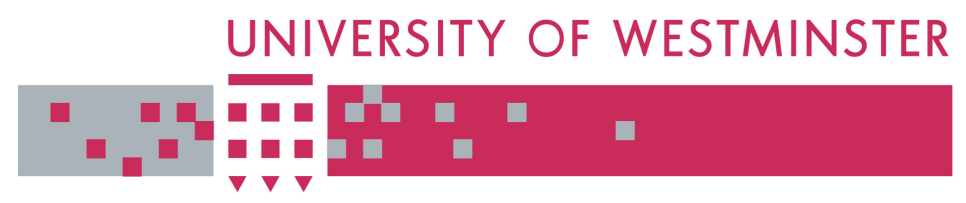

\title{
WestminsterResearch
}

http://www.wmin.ac.uk/westminsterresearch

\section{Novel folded SRR-loaded waveguide bandpass filters}

Niranchana Suntheralingam

Djuradj Budimir

School of Informatics

Copyright (C) [2008] IEEE. Reprinted from the 2008 IEEE Antennas and Propagation Society International Symposium, Jul 5-11, 2008, San Diego, California. IEEE, Los Alamitos, USA, pp. 1-4. ISBN 9781424420414.

This material is posted here with permission of the IEEE. Such permission of the IEEE does not in any way imply IEEE endorsement of any of the University of Westminster's products or services. Personal use of this material is permitted. However, permission to reprint/republish this material for advertising or promotional purposes or for creating new collective works for resale or redistribution to servers or lists, or to reuse any copyrighted component of this work in other works must be obtained from the IEEE. By choosing to view this document, you agree to all provisions of the copyright laws protecting it.

The WestminsterResearch online digital archive at the University of Westminster aims to make the research output of the University available to a wider audience. Copyright and Moral Rights remain with the authors and/or copyright owners.

Users are permitted to download and/or print one copy for non-commercial private study or research. Further distribution and any use of material from within this archive for profit-making enterprises or for commercial gain is strictly forbidden.

Whilst further distribution of specific materials from within this archive is forbidden, you may freely distribute the URL of the University of Westminster Eprints (http://www.wmin.ac.uk/westminsterresearch).

In case of abuse or copyright appearing without permission e-mail wattsn@wmin.ac.uk. 


\title{
Novel Folded SRR-Loaded Waveguide Bandpass Filters
}

\author{
S. Niranchanan, and D. Budimir \\ Wireless Communications Research Group, University of Westminster, \\ 115 New Cavendish Street, London W1W 6UW, United Kingdom \\ Email: d.budimir@wmin.ac.uk
}

\section{Introduction}

Modern wireless communication systems require low cost, mass producible and low dissipation loss microwave and millimetre-wave components such as filters, diplexers, multiplexers and antenna filters. As broadband communication systems are becoming popular, wide band operation becomes an important property for microwave components. The latest interest in implementation of broadband wireless communication systems is conditioned by the set of considerable advantages of such systems over its wired technology analogues. The wider applications demand fulfilment of the new requirements for the systems performance and parameters. This leads to the permanent necessity for the improvement of various components.

The steady growth in commercial interest in microwave and millimeter wave systems such as High data rate line of sight links (LOS), high data rate Wireless Personal Area Networks (WPAN), point to multipoint links, 24 GHz UWB Radar Sensors, and $77 \mathrm{GHz}$ Automotive Radar has provided a significant challenge to conventional microwave circuits and their design methodologies. High performance narrow-band bandpass filters having a low insertion loss, compact size, wide stopband and a high selectivity are important for next-generation wireless and cellular communications, and broadband microwave communication systems. At present most filters at microwave and mm-wave frequencies are produced either in waveguide (air-filled metal pipe, dielectric-filled or micromachined air-filled) with high associated machining costs, image guide and non-radiative dielectric guide with high associated loss $[1,2,3]$ ) or using dielectric resonators although they are less compatible with modern MMIC technology, especially when one is concerned about convenient and efficient integration with active devices or using planar technologies (microstrip, suspended substrate stripline and coplanar waveguide) $[4,5]$.

The microwave and mm-wave applications require wireless communication subsystems in order to transform signals so that they reach the destination with the level, sufficient for proper reception. The high-performance filters are required for separating frequencies in diplexers and multiplexers. They should possess such important properties as low insertion loss and low distortion level along with their compact size realisation. 
Over the past few decades rectangular waveguides have been a sustainable solution used to design robust, low loss and high power circuits at microwave and millimeter-wave frequencies. In the filter structures, which are viable to meet requirements of the modern technology [6], reduction of the physical size has become one of the primary goals. Recently proposed concepts of left-handed medium (LHM) have become the subject of extensive investigations due their capability to provide novel unconventional properties to different propagation media [7]. This approach makes use of the left-handed medium created by a novel type of resonance elements, FSRRs in combination with the thin metal wireline [8]. These are printed on the dielectric slab, which is then inserted into the plane of symmetry of the rectangular waveguide. These structures are able to alter the electromagnetic boundary conditions of the surface and prohibit propagation of signal in a certain frequency band. Thus, the traditional miniaturization techniques, which commonly employ dielectric-filled waveguides with standard dimensions bound to the wavelength $(\lambda)$, may be enhanced to achieve more compact high-performance waveguide components. In this research we make use of the left-handed properties imposed by the novel FSRRs in order to achieve miniaturization of rectangular waveguide filters.

This paper therefore proposes for replacement of the conventional section of rectangular waveguide E-plane filters with a FSRR filter structure, consists of a periodic cascade of metal septa. It maintains the low-cost and mass-producible characteristics of split-block metal insert E-plane technology.

\section{FSRR-Loaded Waveguide Bandpass Filter}

The proposed metamaterial based Folded SRR (FSRR) loaded waveguide filter is shown in Fig. 1. The proposed filter structure is constructed of the transmission line loaded in the metal-dielectric slab, which conveniently facilitates the FSRR filter with the metal septa. The FSRRs are presented on a single side of the dielectric block and is characterised by the following parameters. The dielectric which has relative dielectric permittivity of 2.2 , thickness of $0.51 \mathrm{~mm}$ supports this FSRR filter with the metallisation thickness of $0.017 \mathrm{~mm}$.

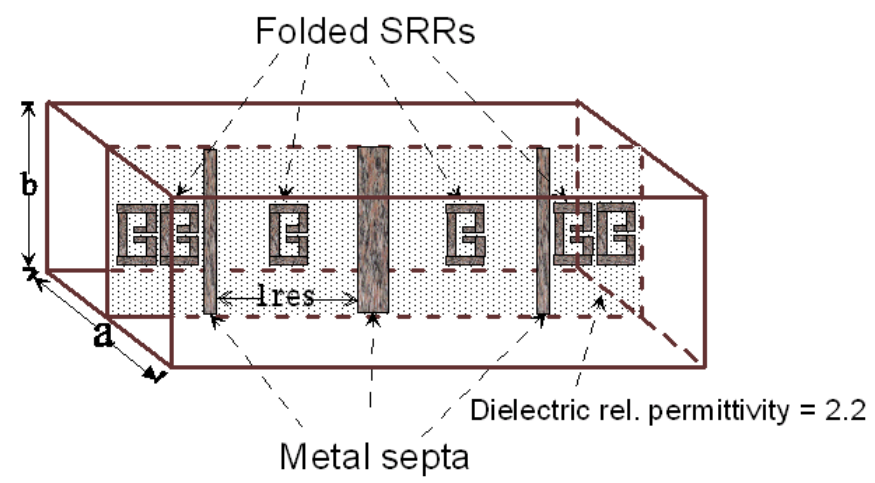

Fig. 1. Configuration of the proposed FSRR loaded bandpass filter 
Standard rectangular waveguide of WG-16 $(\mathrm{a}=22.86 \mathrm{~mm}, \mathrm{~b}=10.16 \mathrm{~mm})$ has been used as a housing to fit the $0.51 \mathrm{~mm}$ thick dielectric slab having total structure length of $51 \mathrm{~mm}$ and $\mathrm{l}_{\text {res }}$ of $14 \mathrm{~mm}$. Simulated insertion and return losses of the proposed bandpass filter are shown in Fig. 2.

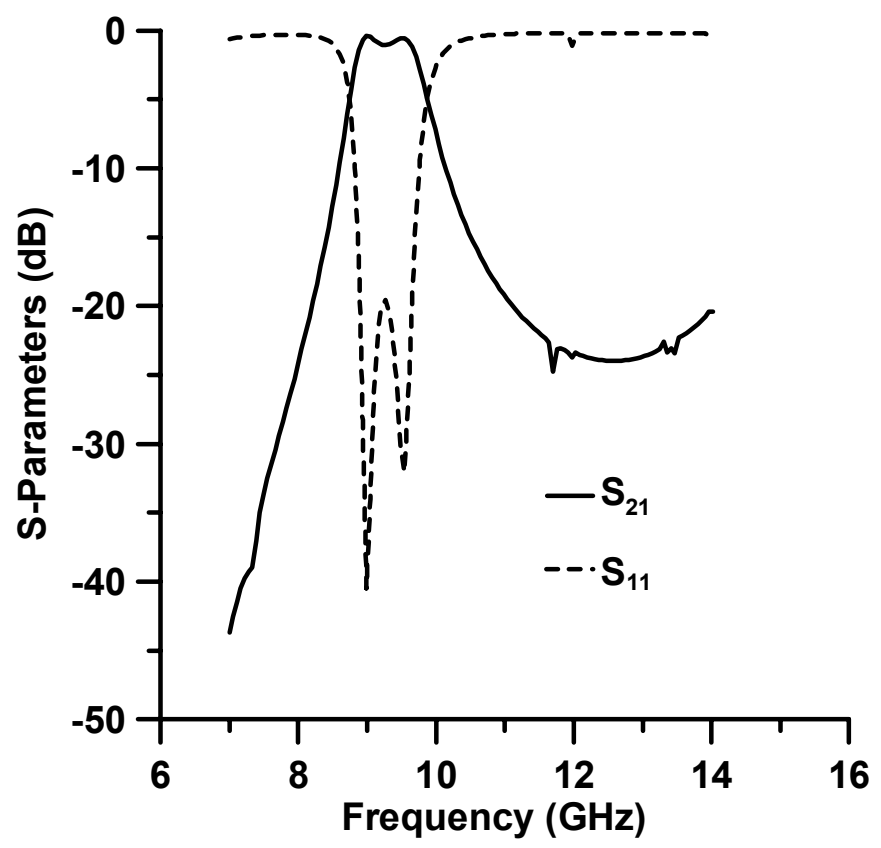

Fig. 2. Simulated Insertion and Return losses of the proposed FSRR-loaded bandpass filter

\begin{tabular}{|l|c|}
\hline \multicolumn{2}{|c|}{ Parameters (mm) } \\
\hline WG inner dimensions (WG16) & $22.86^{\star}$ 10.16 \\
\hline Total structure length & 51 \\
\hline Dielectric slab length & 51 \\
\hline Dielectric thickness & 0.51 \\
\hline Metal septa length & $1.2,3,1.2$ \\
\hline Ires & 12 \\
\hline Metallization thickness & 0.0017 \\
\hline Distance between the two & 1.28 \\
Folded SRRs on the side & \\
\hline
\end{tabular}

Table I. Dimensions of the proposed FSRR-loaded bandpass filter 
The electromagnetic analysis of the proposed metamaterial based FSRR filter structure is conveniently based on the finite element method $\left(\mathrm{HFSS}^{\mathrm{TM}}\right)$ [9]. In order to derive a design procedure for the proposed type of the filter, the propagation characteristics of the slow wave, such as the guided wavelength or phase velocity need to be determined. These in turn are determined by geometrical parameters, namely the gaps and the lengths. The gaps can be chosen arbitrarily. The periodicity lengths, together with the chosen gaps will effectively determine the wavelength; this in turn should determine the length $1_{\text {res. }}$ Dimensions of the proposed FSRR filter at $9.45 \mathrm{GHz}$ are given in Table I.

\section{Conclusion}

A novel class of E-Plane, low-cost, compact and metamaterial-based filter structure using FSRRs for microwave, millimeter wave applications has been proposed. The proposed FSRR-loaded waveguide bandpass filter has been designed and simulated at $9.45 \mathrm{GHz}$. The structure can be easily realized with a single metal insert within a rectangular waveguide. This kind of filters can be found in applications particularly in the mm-wave range circuits, e.g. in diplexers and multiplexers.

\section{References:}

[1]V. Postoyalko, and D. Budimir, "Design of Waveguide E-plane Filters with All-Metal Inserts by Equal-Ripple Optimization," IEEE Trans. Microwave Theory \& Tech., vol. MTT-42, pp. 217-222, February 1994

[2]V. G. Veselago, "The electrodynamics of substances with simultaneously negative values of $\varepsilon$ and $\mu$," Soviet Phys. Uspekhi, vol. 10, no. 4, pp. 509-514, Jan.-Feb. 1968

[3]J.B. Pendry, A.J. Holden, D.J. Robbins, and W.J. Stewart, "Magnetism from conductors and enhanced nonlinear phenomena," IEEE Trans. Microwave Theory \& Tech., vol. 47, no. 11, pp. 2075-2084, November 1999

[4]A. Shelkovnikov, N. Suntheralingam, and D. Budimir, "Novel SRR Loaded Waveguide Bandstop Filters," IEEE AP-S/URSI Int. Symp., Albuquerque, USA, July 2006

[5]D. Budimir, "EPFIL-Waveguide E-plane Filter Design", Software and User Manual, ISBN 1-58053-083-4, Artech House Books, 1999

[6]D. Budimir, "Generalized Filter Design by Computer Optimization", ISBN 089006-579-9, Artech House Books, 1998

[7]H. Contopanagos, N. G. Alexopoulos, and E. Yablonvitch, "High-Q Ractangular Cavities and Waveguide Filters Using Periodic Metalo-Dielectric Slabs”, IEEE MTT-S Digest, pp 1539-1542, 1998

[8]Yan Li, L. Ran, H. Chen, J. Huangfu, X. Zhang, K. Chen, T. M. Grzegorczyk, and J. A. Kong, "Experimental Realization of a One-Dimensional LHMRHM Resonator," IEEE Trans. Microwave Theory \& Tech., vol. 53, no. 4, pp. 1522-1526, April 2005

Ansoft HFSS, Ansoft Technologies, 2005 